\title{
CURVATURE GROUPS OF A HYPERSURFACE
}

\author{
SAMUEL I. GOLDBERG ${ }^{1}$
}

\begin{abstract}
A cochain complex associated with the vector 1-form determined by the first and second fundamental tensors of a hypersurface $M$ in $E^{n+1}$ is introduced. Its cohomology groups $H^{P}(M)$, called curvature groups, are isomorphic with the cohomology groups of $M$ with coefficients in a subsheaf $\delta_{R}$ of the sheaf $\delta$ of closed vector fields on $M$. If $M$ is a minimal variety, the same conclusion is valid with $\delta_{R}$ replaced by a sheaf of harmonic vector fields. If the Ricci tensor is nondegenerate the $H^{p}(M)$ vanish. If $\delta_{R} \neq \varnothing$, and there are no parallel vector fields, locally, the $H^{p}(M)$ are isomorphic with the corresponding de Rham groups.
\end{abstract}

1. Introduction. A cochain complex associated with the Levi-Civita connection of a Riemannian manifold $M$ was studied in [1], and it was shown that its cohomology groups, called curvature groups, are isomorphic with the cohomology groups of $M$ with coefficients in a sheaf of germs of infinitesimal homothetic transformations of $M$. This extended the main result of [3] concerning locally flat manifolds. Subsequent work of I. Vaisman [4] suggests that if one considers the vector 1 -form $\sigma$ determined by the first and second fundamental tensors of a hypersurface $M$ in the Euclidean space $E^{n+1}$ rather than the solder form of the bundle of frames over $M$, a cochain complex with similar properties may be defined. The curvature groups, that is, the cohomology groups $H^{p}(M)$ of this complex turn out to be isomorphic with the cohomology groups of $M$ with coefficients in a subsheaf $\delta_{R}$ of the sheaf $\delta$ of 'closed' vector fields on $M$, and if $M$ is a minimal variety then the same conclusion prevails, but $\delta_{R}$ may then be replaced by a sheaf of harmonic vector fields.

Curvature is introduced by means of the integrability conditions of the Pfaff system defining the elements of $\delta$. If the Ricci tensor is nondegenerate everywhere, then the curvature groups vanish. In particular, if $M$ is an Einstein space with nonvanishing scalar curvature, the $H^{p}(M)$ are trivial. (If $n \geqslant 3$ or if $n=2$ and $M$ has constant sectional curvature $\neq 0$, then $M$ is a hypersphere.) Finally, if $\delta_{R} \neq 0$, and there are no parallel vector fields, locally, the $H^{p}(M)$ are isomorphic with the corresponding de Rham groups of $M, p=1, \ldots, n$.

2. The cochain complex of tensorial $p$-jet forms. To avoid unnecessary duplication the notation and terminology of [1] will be employed unless otherwise indicated.

Received by the editors November 11, 1975.

AMS (MOS) subject classifications (1970). Primary 53B25, 55B30; Secondary 53A10, 53B20.

Key words and phrases. Jet forms, curvature groups, closed vector fields, sheaf cohomology.

${ }^{1}$ Research partially supported by the National Science Foundation. 
Let $M$ be an $n$-dimensional Riemannian manifold locally isometrically imbedded in $E^{n+1}$ with the Euclidean metric. In terms of the cartesian coordinates of $M$ let $\sigma_{i j}$ denote the coefficients of the second fundamental form of $M$. Then the curvature of $M$ is given by the Gauss equations

$$
R_{i j k 1}=\sigma_{j k} \sigma_{i l}-\sigma_{j l} \sigma_{i k}
$$

and the Codazzi equations are

$$
\nabla_{k} \sigma_{i j}=\nabla_{j} \sigma_{i k}
$$

If the mean curvature of the hypersurface vanishes, that is, if $g^{i j} \sigma_{i j}=0$, where $g$ is the metric tensor of $M$, then $M$ is called a minimal hypersurface or a minimal variety of $E^{n+1}$. (The summation convention is employed throughout.) It is well known that a totally geodesic hypersurface is a minimal variety.

Consider the vector 1 -form $\sigma$ given by

$$
\sigma^{i}=\sigma_{j}^{i} d x^{j}, \quad \sigma_{j}^{i}=g^{i r} \sigma_{r j}
$$

From (2)

$$
\nabla \sigma=0 .
$$

Let $L^{p}$ denote the $\mathfrak{F}$-module defined by the pairs $(\lambda, \alpha)$, where $\lambda$ is a vectorvalued $p$-form and $\alpha$ is a scalar $p$-form on $M$ for $p=1, \ldots, n$ (see [1]). For each $p$, an operator $D^{p}: L^{p} \rightarrow L^{p+1}$ is defined by

$$
D^{p}(\lambda, \alpha)=(\nabla \lambda-\sigma \wedge \alpha, d \alpha) .
$$

If $(\lambda, \alpha)$ is $D^{p}$-closed, then $\alpha$ is $d$-exact, locally, and $\nabla^{2} \lambda=(\nabla(\nabla \lambda))$ is zero. Note that $\left(\nabla^{2} \lambda\right)^{i}=-\Omega_{j}^{i} \wedge \lambda^{j}$, where the $\Omega_{j}^{i}=R_{j k l}^{i} d x^{k} \wedge d x^{l}$ are the curvature forms of the Riemannian connection.

Let $\tilde{L}^{p}$ denote the submodule of $L^{p}$ defined by those pairs $(\lambda, \alpha)$ with $\nabla^{2} \lambda=0$. Observe that $\tilde{L}^{p}=L^{p}$ for $p=n-1, n$; moreover, the pairs $(\sigma \wedge \varphi, \alpha) \in \tilde{L}^{p}$ for any scalar $(p-1)$-form $\varphi$ and $p$-form $\alpha$ on $M$. From (3), $D^{p}: \tilde{L}^{p} \rightarrow \tilde{L}^{p+1}$, and $D^{p+1} D^{p}=0$.

In the sequel, we shall occasionally write $D$ for $D^{p}$.

Consider the cochain complex

$$
\tilde{L}=\left(\bigoplus_{p=0}^{n} \tilde{L^{p}}, D^{p}\right),
$$

and assume that the Poincare lemma holds for $D$, that is, on a star-shaped region (open ball) in $R^{n}$, every $D$-closed element of $\tilde{L}^{p}, p>0$, is $D$-exact. In the sequel, we consider only complexes for which the Poincare lemma is valid, and denote them again by $\tilde{L}$. This is certainly the case if $M$ is locally flat. If rank $\sigma \geqslant 2$, that is, if the type number of $M$ is $\geqslant 2$, the Poincare lemma also holds for the subcomplex of $\tilde{L}$ obtained by considering only the pairs $(\sigma \wedge \varphi, \alpha)$.

The cohomology groups

$$
H^{p}(\tilde{L})=\operatorname{ker} D^{p} / \operatorname{im} D^{p-1}, \quad p=1, \ldots, n,
$$


will be called the curvature groups of the hypersurface. We shall also write $H^{p}(M)$ for $H^{p}(\tilde{L})$, and denote ker $D^{0}$ by $H^{0}(M)$.

A graded ring structure is defined on $\tilde{L}$ as in [1], and $D$ turns out to be a derivation on this ring.

3. The sheaf of germs of $c$-fields. Since the coefficients of the second fundamental form are symmetric, the system of first order partial differential equations

$$
\nabla_{j} X^{i}=f \sigma_{j}^{i},
$$

where $X=X^{i} \partial / \partial x^{i}$ is a vector field on $M$ and $f$ is a smooth function, gives rise to a closed 1-form $\xi=\xi_{i} d x^{i}, \xi_{i}=g_{i k} X^{k}$. Hence, by the Poincaré lemma, $\xi$ is the gradient of a function, locally. If $M$ is a minimal variety, then $X$ is a harmonic vector field. The vector fields characterized as solutions of (4) will be called closed vector fields, or simply c-fields. They form an additive abelian group $S$ but not an $\mathfrak{F}$-module.

The integrability conditions of (4) yield

$$
X^{r} R_{r j k}^{i}=\sigma_{j}^{i} \nabla_{k} f-\sigma_{k}^{i} \nabla_{j} f
$$

Contracting gives

$$
X^{r} R_{r j}=\sigma_{j}^{r} \nabla_{r} f-\sigma_{r}^{r} \nabla_{j} f
$$

so if $M$ is a minimal variety, $X^{r} R_{r j}=\sigma_{j}^{r} \nabla_{r} f$.

We are particularly interested in the case where $f=$ constant since this yields an interpretation of the curvature groups in terms of the sheaf cohomology of the complex $\tilde{L}$. Thus, from $(5), \iota(X) \Omega=0$, and this is satisfied if $M$ is locally flat. On the other hand, if the Ricci tensor is nondegenerate everywhere, there are no nontrivial solutions of (4).

4. Cohomology with coefficients in the sheaf of germs of $c$-fields. Let $S_{R}$ be the subspace of $c$-fields characterized as solutions of (4) with $f=c$ (constant). There is a monomorphism $i: S_{R} \rightarrow \tilde{L}^{0}$ defined by $i(X)=(X, c)$. Let $\delta_{R}$ denote the sheaf of germs of vector fields associated with $S_{R}$, and let $\mathcal{L}^{p}, p \geqslant 0$, denote the sheaves of germs associated with the $\mathfrak{F}$-modules $\tilde{L}^{p}$. The mapping $D: \tilde{L}^{p} \rightarrow \tilde{L}^{p+1}$ induces a mapping $\mathfrak{L}^{p} \rightarrow \varrho^{p+1}$ which is again denoted by $D$. We then have a sequence of sheaf homomorphisms

$$
0 \rightarrow \delta_{R} \stackrel{i}{\rightarrow} \mathcal{L}^{0} \stackrel{D}{\rightarrow} \mathcal{L}^{1} \stackrel{D}{\rightarrow} \ldots \stackrel{D}{\rightarrow} \mathcal{L}^{n} \rightarrow 0
$$

which is exact. In fact, if $(X, f) \in \mathcal{L}^{0}$ and $D(X, f)=(\nabla X-f \sigma, d f)=0$, then $\nabla X=c \sigma$, where $f=$ const $=c$. Hence, $(X, f)=i(X)$. Exactness at $\varrho^{p}$ for $p$ $>0$ is a consequence of the Poincaré lemma for $D$. The $\mathcal{L}^{p}$ for $p=0,1, \ldots, n$ being fine sheaves, the sequence is a fine resolution of $\mathfrak{S}_{R}$. We therefore have the following interpretation of the groups $H^{p}(M)$.

THEOREM 1. The curvature groups $H^{p}(M)$ of a hypersurface $M$ are isomorphic with the cohomology groups $H^{p}\left(M, \delta_{R}\right)$ of the hypersurface with coefficients in $\mathcal{S}_{R}, 0 \leqslant p \leqslant n$. 
COROLlARY 1. The curvature groups of a hypersurface with nondegenerate Ricci tensor everywhere vanish.

Thus, if the Ricci tensor is nondegenerate everywhere then a $D$-closed 1 form can be expressed as $(-f \sigma, d f)$.

COROLlARY 2. The curvature groups of a hypersurface $M$ with an Einstein metric of nonzero scalar curvature are trivial.

If $n \geqslant 3$ or if $n=2$ and $M$ has constant sectional curvature $\neq 0$, then $M$ is a hypersphere.

If the hypersurface is minimal, in particular, if it is totally geodesic, the curvature groups have the following interpretation.

THEOREM 2. The curvature groups of a minimal variety are isomorphic with the cohomology groups of the hypersurface with coefficients in a sheaf of germs of its harmonic vector fields.

COROLlARY. The curvature groups of a totally geodesic hypersurface are isomorphic with the cohomology groups of the hypersurface with coefficients in the sheaf of germs of its parallel vector fields.

Note that if $M$ is umbilical, then $\sigma$ is proportional to the solder form $\eta$ of the bundle of frames over $M$, that is, $\sigma=\mu \eta$ for some smooth function $\mu$. The above theory is then a consequence of that developed in [1].

THEOREM 3. The curvature groups of an umbilical hypersurface $M$ of $E^{n+1}$, with no flat points, are isomorphic with the curvature groups of $M$ with coefficients in the sheaf of germs of homothetic s-fields.

Since by the Codazzi equations, $\mu$ is actually a constant, it follows that if $M$ is complete and connected and $\mu \neq 0$, then $M$ is a hypersphere. If $\mu=0$, then under the conditions, $M$ is a hyperplane.

5. The complex of vector forms of the type $\sigma \wedge \varphi$. Let $\Phi^{p}$ be the $\mathfrak{F}$-module of vector-valued forms expressible as $\sigma \wedge \varphi$ where $\varphi$ is a scalar-valued $(p-1)$-form. By (3), $\nabla(\sigma \wedge \varphi)=-\sigma \wedge d \varphi$. Thus, $\nabla(\nabla(\sigma \wedge \varphi))=0$. Consider the complex of forms $\Phi=\left(\oplus_{p=1}^{n} \Phi^{p}, \nabla^{p}\right)$ where $\nabla^{p}=\left.\nabla\right|_{\Phi^{p}}$, and let $H^{p}(\Phi)=\operatorname{ker} \nabla^{p} /$ im $\nabla^{p-1}$ denote its $p$ th cohomology group. Define $H^{1}$ $=\operatorname{ker} \nabla^{1}$. Assuming rank $\sigma \geqslant 2$, the chain map $\varphi \rightarrow \sigma \wedge \varphi$ establishes a bijection of the module of $p$-forms on $M$ with $\Phi^{p+1}$ for $p=0,1, \ldots, n-1$. Clearly $d$-closed forms are mapped into $\nabla$-closed forms and $d$-exact forms into $\nabla$-exact forms. Thus, the $p$-dimensional de Rham groups of $M$ are isomorphic with the groups $H^{p+1}(\Phi)$.

A multiplication "o" between the elements of $\Phi$ is defined by

$$
\sigma \wedge \varphi \circ \sigma \wedge \psi=\sigma \wedge \varphi \wedge \psi \in \Phi^{p+q-1},
$$

where $\varphi$ and $\psi$ are scalar $(p-1)$ - and $(q-1)$-forms, respectively. It is an easy consequence that

$$
\begin{aligned}
& \sigma \wedge \varphi \circ \sigma \wedge \psi \\
& \nabla\left(\sigma \wedge(-1)^{p q} \sigma \wedge \psi \circ \sigma \wedge \varphi,\right. \\
&\nabla \circ \wedge \psi)=\nabla(\sigma \wedge \varphi) \circ \sigma \wedge \psi+(-1)^{p-1} \sigma \wedge \varphi \circ \nabla(\sigma \wedge \psi) .
\end{aligned}
$$


Hence, $\Phi$ is a graded ring and $\nabla$ is a derivation on $\Phi$. Moreover, the cohomology ring of $\Phi$ is isomorphic with the de Rham cohomology ring of $M$.

THEOREM 4. Let $M$ be a hypersurface of $E^{n+1}$. If the system (4) has a solution for some constant $c \neq 0$ but no nontrivial solution for $c=0$, then the curvature groups $H^{p}(M)$ are isomorphic with the p-dimensional de Rham groups, $1 \leqslant p$ $<n$.

The proof is an easy consequence of Theorem 1 , since by hypothesis the sheaf $\delta_{R}$ is isomorphic with the sheaf of real constants.

REMARKs. (a) If $M$ is locally convex the conditions of the theorem do not hold, as one sees from (6) and [2, p. 124].

(b) If $\nabla X=c \sigma$ has a solution for some $c \neq 0$, then it has a solution for every $c \neq 0$. We therefore have a sequence of homomorphisms

$$
0 \stackrel{i}{\rightarrow} S_{R} \stackrel{\nabla}{\longrightarrow} \Phi^{1} \stackrel{\nabla}{\longrightarrow} \Phi^{2} \stackrel{\nabla}{\longrightarrow} \cdots \stackrel{\nabla}{\longrightarrow} \Phi^{n} \stackrel{\nabla}{\longrightarrow} 0 .
$$

Let $\mathfrak{F}^{p}, 1 \leqslant p \leqslant n$, be the sheaves of germs associated with $\Phi^{p}$. Then, the above sequence induces the sequence of sheaf homomorphisms

$$
0 \stackrel{i}{\rightarrow} \mathcal{S}_{R} \stackrel{\nabla}{\longrightarrow} \mathfrak{F}^{1} \stackrel{\nabla}{\longrightarrow} \mathfrak{F}^{2} \stackrel{\nabla}{\longrightarrow} \cdots \stackrel{\nabla}{\longrightarrow} \mathfrak{F}^{n} \stackrel{\nabla}{\longrightarrow} 0
$$

so under the assumptions of Theorem 4 this sequence is exact provided rank $\sigma \geqslant 2$. Since the $\widetilde{F}^{p}$ are fine sheaves, the sequence (7) is a fine resolution of $\delta_{R}$, and hence the groups $H^{p+1}(\Phi)$ are isomorphic with the groups $H^{p}\left(M, \varsigma_{R}\right)$ for $1 \leqslant p<n$.

(c) If $M$ is a hypersurface locally isometrically imbedded in an $(n+1)$ dimensional space of nonzero constant curvature, the above theory may again be applied.

(d) The curvature groups can be generalized as follows: Let $M$ be a differentiable manifold endowed with a field of endomorphisms of its tangent spaces or, equivalently, with a vector 1 -form $\sigma$. If a linear connection $\nabla$ with $\nabla \sigma=0$ is chosen, a cochain complex $\tilde{L}$ and its 'curvature groups' can be constructed as in $\$ 2$. One then seeks interesting corresponding sheaves. Many geometrical structures are then included in this scheme. (The real difficulty lies in obtaining a Poincaré lemma for $D$.)

Problems. (A) In the given scheme do the curvature groups depend on the connection chosen?

(B) Can one find a suitable generalization to arbitrary $G$-structures?

\section{BIBLIOGRAPHY}

1. S. I. Goldberg and N. C. Petridis, The curvature groups of a pseudo-Riemannian manifold, J. Differential Geometry 9 (1974), 547-555.

2. S. I. Goldberg, Curvature and homology, Pure and Appl. Math., vol. 11, Academic Press, New York and London, 1962. MR 25 \#2537.

3. I. Vaisman, The curvature groups of a space form, Ann. Scuola Norm. Sup. Pisa (3) 22 (1968), 331-341. MR 37 \#6955.

4. - The curvature groups of a hypersurface in the Euclidean space, Acta Math. Acad. Sci. Hungar. 23 (1972), 21-31. MR 47 \#2521. 